\title{
Course organisers' conference 5 February 1996
}

\author{
John Cox
}

This was only the second conference for MRCPsych course organisers to be held in the UK or the Republic of Ireland. The morning session (introduced by the President) considered the College requirements for an approved MRCPsych course, and in the afternoon the nature and extent of multi-professional education and training were discussed.

The Dean said at the outset that there was no specific statement at present of the criteria for a College-approved MRCPsych course, and he hoped that the day would clarify the likely content of such objectives. The nature of Educational Contracts, a concern for 'value for money' as well as the more public educational strategy have underlined the need for these objectives to be clarified.

He welcomed also the opportunity to discuss interprofessional education, noting the interest of the NHS Training Division and the Centre for the Advancement of Interprofessional Education, as well as articles in the Journal of Medical Education on these themes.

Dr Lynn Drummond opened the conference by outlining the St George's MRCPsych course. This comprehensive course includes Supervised Research, Mock Clinical Examinations and Essay Practice (two per term), as well as practice in multiple choice questions sponsored by the pharmaceutical industry: In addition there was a Mental Health Act training day and a special course on Sexual Dysfunction. The course lasted for six 10-week terms over two years. Attendance for trainees was compulsory and failure to attend penalised. The lecturers recetved compulsory feedback and Dr Drummond emphasised that the teaching was based very closely on the MRCPsych curriculum.

Professor Shôn Lewis from the University of Manchester emphasised the need for an MRCPsych course to include critical appraisal of the literature, and suggested the use of an academic logbook. He said that the volume of knowledge expected by the College at the present time was "too much". There was a need for coordination. He advocated compulsory feedback, league tables for examination success and thought that the Central Approval Panel might have a greater role in the inspection of MRCPsych courses.

Dr Jeremy Bolton, Chairman of the Psychiatric Tutors' Sub-Committee, emphasised that MRCPsych courses also provided peer support and said there was need for the educational training of teachers. He said that an MRCPsych course was not intended to provide practical clinical teaching nor a 'Control and Restraint' course, but to provide theoretical knowledge.

Dr Gary Sullivan (CTC) described difficulties where there was no formal MRCPsych course available but only a taught Master's degree.

In discussion there was an emerging consensus that the objectives of an MRCPsych course should include an indication of purpose, content and duration, together with mandatory core contents such as risk assessment and Mental Health Act training. A statement that a purpose of the MRCPsych course was to prepare trainees to take the MRCPsych examination would be valuable. An approved course would be expected to be based on the MRCPsych curriculum and the examination requirements. Educational methods were likely to be those associated with adult learning, to be iterative, problem-solving, and to involve small group participation. The teaching qualification of teachers should be considered.

The afternoon session was introduced by Dr John Robertson and Dr Jeremy Bolton who reported on their survey of course organisers with regard to the current attitudes and practice in the field of multi-professional teaching. They showed that non-psychiatrists were already participating as teachers on MRCPsych courses, more especially in the field of psychology, basic sciences and statistics; the proportion of such teaching ranging from $20-30 \%$. None of the respondents indicated that social work teachers were presently involved. Furthermore, the respondents indicated that students from other professional backgrounds - nursing, social work and psychology - only very infrequently took part in seminars.

In the discussion that followed it was questioned whether students from other disciplines would welcome a chance to participate in an MRCPsych course designed to assist a post- 
graduate doctor to obtain the Professional Examination of a Royal College. Others present thought that the experience of interprofessional learning would facilitate greater team working subsequently.

Ms Janis Stout (British Association of Social Workers) said that they were currently celebrating a hundred years of health-related social work and firmly advocated multi-professional and interagency working and training, a theme also supported by Dr Paula Roberts, Lecturer in Nursing in North Staffordshire. She said there was a need to work with 'agents for change' on an informal basis and asked those present to consider what was the end product of their training programmes.

Dr Til Wykes of the Institute of Psychiatry challenged the audience by asking "What do psychiatrists want to be?". Do they wish to appreciate the role of psychologists or to participate in joint working? Where are the 'edges' of the role such as drug prescription, psychological therapies, Care Planning and Mental Health Act legislation? Why was it that doctors rarely took part in multi-professional training?

With regard to psychology teaching she said there was a need for greater clarity as to what should be taught, to what standard and who should teach. There were few clinical psychologists and teaching from these scarce professionals, though desirable, might be difficult to obtain.

In the discussion, the role of psychiatrists was emphasised with regard to assessment and ability to recognise the indications for, and the practice of, psychological and social treatments, as well as biological treatments.

The day was valued by participants and highlighted the need for collaboration between MRCPsych course organisers and MSc course organisers, as well as for greater collaboration with college tutors responsible for formative assessments.

In general, multi-professional teaching happened but multi-professional learning was rare. It is hoped that the report of this day will stimulate further debate and discussion about the undoubtedly important matters described above. There was consensus that an annual meeting of MRCPsych course organisers would be necessary and was likely to be valuable.

John L. Cox, Dean, The Royal College of Psychiatrists, and Professor of Psychiatry, Keele University, Staffordshire 